UEDGE Code Comparisons with DIII-D Boloneter DATA

Jennifer M. Daniel

January 1995

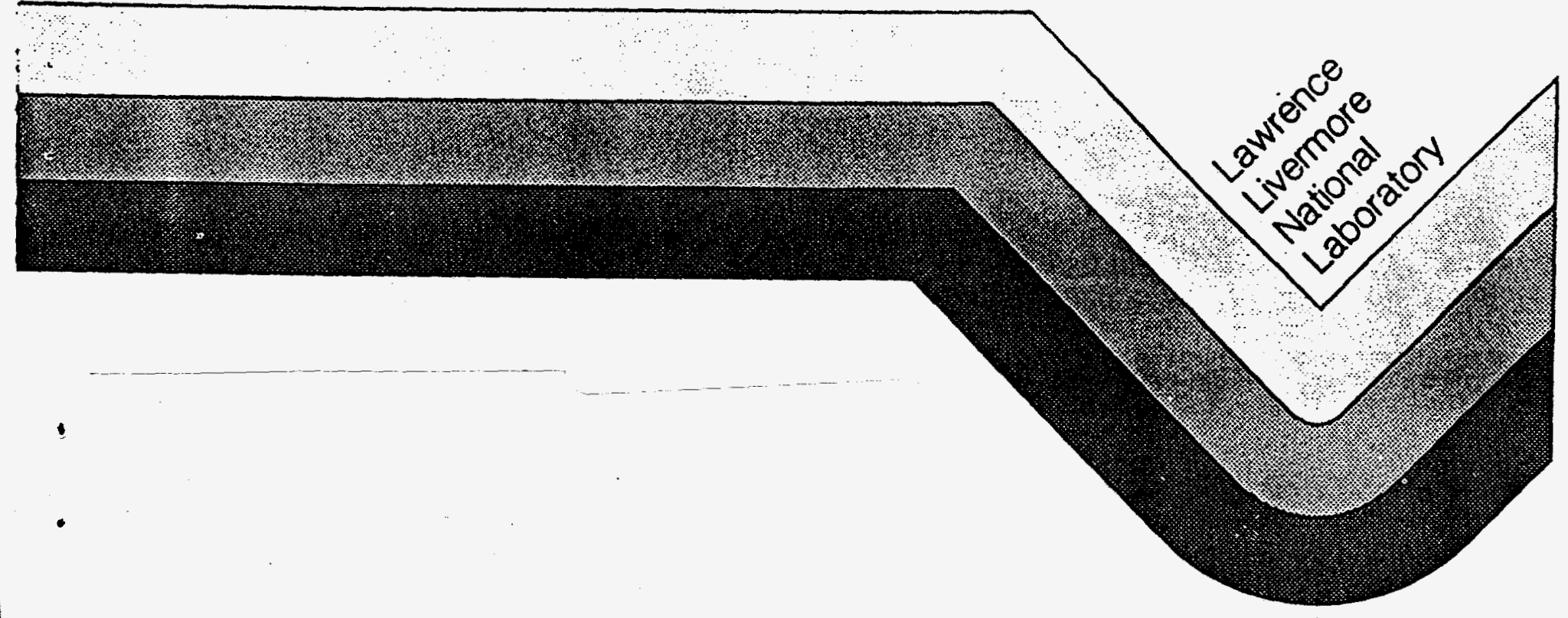




\section{DISCLAIMER}

This report was prepared as an account of work sponsored by an agency of the United States Government. Neither the United States Government nor any agency thereof, nor any of their employees, make any warranty, express or implied, or assumes any legal liability or responsibility for the accuracy, completeness, or usefulness of any information, apparatus, product, or process disclosed, or represents that its use would not infringe privately owned rights. Reference herein to any specific commercial product, process, or service by trade name, trademark, manufacturer, or otherwise does not necessarily constitute or imply its endorsement, recommendation, or favoring by the United States Government or any agency thereof. The views and opinions of authors expressed herein do not necessarily state or reflect those of the United States Government or any agency thereof. 


\section{DISCLAIMER}

Portions of this document may be illegible in electronic image products. Images are produced from the best available original document. 


\title{
UEDGE CODE COMPARISONS WITH DIII-D BOLOMETER DATA*
}

\author{
Jennifer M. Daniel \\ Lawrence Livermore National Laboratory \\ Livermore, California 94550
}

December 14, 1994

*This work was performed under the auspices of the U.S. Department of Energy by Lawrence Livermore National Laboratory under contract number W7405-ENG-48, also by General Atomics under contract number DE-AC0389ER51114. 


\title{
UEDGE Code Comparisons With DIII-D Bolometer Data
}

\author{
Jennifer M. Daniel \\ Athens State College \\ Magnetic Fusion Energy Division
}

\begin{abstract}
*
This paper describes the work done to develop a bolometer post processor that converts volumetric radiated power values taken from a UEDGE solution, to a line integrated radiated power along chords of the bolometers in the DIII-D tokamak. The UEDGE code calculates plasma physics quantities, such as plasma density, radiated power, or electron temperature, and compares them to actual diagnostic measurements taken from the scrape off layer ( SOL ) and divertor regions of the DIII-D tokamak. Bolometers are devices measuring radiated power within the tokamak. The bolometer interceptors are made up of two complete arrays, an upper array with a vertical view and a lower array with a horizontal view, so that a two dimensional profile of the radiated power may be obtained. The bolometer post processor stores line integrated values taken from UEDGE solutions into a file in tabular format. Experimental data is then put into tabular form and placed in another file. Comparisons can be made between the UEDGE solutions and actual bolometer data. Analysis has been done to determine the accuracy of the plasma physics involved in producing UEDGE simulations.
\end{abstract}

*This work was performed under the auspices of the U.S. Department of Energy by Lawrence Livermore National Laboratory under contract number W7405-ENG-48, also by General Atomics under contract number DE-AC0389ER51114. 
Prepared in partial fulfillment of the requirements of the Science and Engineering Research Semester under the direction of Max Fenstermacher and Gary Porter, Research Mentors, in the Lawrence Livermore National Laboratory.

* This research was supported in part by an appointment to the U. S. Department of Energy Science and Engineering Research Semester (hereinafter called SERS) program administered by LLNL under Contract W7405-Eng-48 with Lawrence Livermore National Laboratory.

By acceptance of this article, the publisher or recipient acknowledges the U. S. Government's right to retain a non-exclusive, royalty-free license in and to any copyright covering this article. 


\section{OUTLINE}

Thesis: Comparisons of UEDGE generated quantities with DIII-D bolometer data provides a method for bench marking the physics models used in UEDGE plasma simulations.

I. Background Information
A. Fusion Energy
B. Tokamaks
C. Bolometers

II. Bolometer Diagnostic
A. Sensor Description
B. Geometry for Viewing
C. Old versus New arrays

III. UEDGE Code
A. Purpose of the UEDGE Code
B. How the UEDGE Code Works
C. How radiated power along bolometer lines of site is
calculated by the UEDGE Code

IV. Bolometer Post Processor
A. Purpose of the Bolometer Post Processor
B. How the Bolometer Post Processor Works
C. How the bolometer Post Processor prepares UEDGE and bolometer data for comparisons

V. Analysis of Shot 82151 and Comparison to UEDGE Simulations
A. Simulation with no impurity radiation
B. Simulation with constant impurity concentration
C. Simulation with impurity transport

VI. Conclusions from Comparisons
A. Impurity Model Needed
B. Carbon Impurity Introductions
C. Physics of Carbon migration

VII. Summary 


\section{UEDGE CODE COMPARISONS WITH DIII-D BOLOMETER DATA}

\section{Introduction}

According to Newton's laws, energy is neither created nor destroyed. However, it is transformed. Power is one form of energy transformation. It is the time rate of change of energy transferred. Some sources for power generation include hydroelectric plants, windmills, solar power, and nuclear power plants. Fusion power, currently being researched and developed, is another source for power generation. The DIII-D experiment at General Atomics in San Diego, California in cooperation with Lawrence Livermore National Laboratory (LLNL), is part of the ongoing research for fusion power. The DIII-D machine uses a toroidal device, called a tokamak, for a magnetic confinement system. This system helps contain a hot ionized gas called plasma, the fourth state of matter. The plasma reaches temperatures of approximately $10-50$ million degrees Kelvin in the center of the tokamak. There are devices on the tokamak called bolometers which measure the heat or power radiated from the plasma. Lawrence Livermore National Laboratory assists General Atomics in their data analysis for DIII-D. LLNL has a code, UEDGE, that simulates the edge tokamak plasma parameters and generates quantities similar to those measured in actual DIIID experiments. Current studies are being done to examine the power radiated within DIII-D and compare this to UEDGE simulations at LLNL. Comparisons of UEDGE generated quantities with DIII-D bolometer data provides a method for bench marking the physics models used in UEDGE plasma simulations. 


\section{Background}

\section{Fusion Energy}

Fusion occurs when two light nuclei combine to form a heavier nucleus. In some reactions the mass of the final nucleus is less than the combined rest masses of the original nuclei. Due to a loss of mass in these fusion reactions, there is also an accompanying release of energy (Serway 1395). The goal for fusion research is to capture this released energy from the reactions, and use it for power generation here on earth. One might ask why we need another source of power generation. Although we now have several sources of power generation, studies show, with a future decrease in availability of fuels, other sources of power generation are needed. Currently, nuclear power plants rely on Uranium as a fuel source, which is not very abundant. However, if a fusion reactor is fully developed, the heavy isotope of hydrogen that is required as fuel, can be found in water.

In water there exists a hydrogen isotope called deuterium also known as heavy hydrogen, having twice the mass of ordinary hydrogen. Deuterium is extracted from the water, and then the atoms are fused in reactions such as:

$$
{ }_{1} \mathrm{H}^{2}+1 \mathrm{H}^{2} \longrightarrow 2 \mathrm{H}^{3}+\mathrm{On}^{1}
$$

The total amount of energy released from each deuterium reaction is around $3.27 \mathrm{MeV}$ (Serway 1396). A similar reaction combining deuterium and tritium, a radioactive hydrogen isotope, produces $17.59 \mathrm{MeV}$ of energy per fusion reaction (Serway 1396). Given the same conditions and temperatures to initiate fusion, the deuteriumdeuterium, DD, reaction is less likely to occur, while in the deuterium-tritium, DT, reaction, which releases more energy, and the atoms are more likely to fuse. Although the DT reaction releases more energy, the DD reaction is favored for two reasons: 1) tritium is radioactive, while deuterium is not, and 2) tritium is not naturally occurring in water like deuterium (Wesson 2). The ultimate goal is to use the DD reaction for a fully developed commercial fusion reactor. 
In combining two atoms, the repulsive force between two charged nuclei must be overcome. To do this; kinetic energy must be supplied to the nuclei by heating the fuel to high temperatures (approximately $4 \times 10^{8} \mathrm{~K}$ for the DD reaction and $4.5 \times 10^{7}$ $\mathrm{K}$ for the DT reaction) (Serway 1398). At such high temperatures, atoms ionize and a plasma forms.

After the plasma forms the problem is a matter of efficiency and containment. More power must be harnessed from the fusion reaction than is put in to heat the fuel and fuse the atoms together; that is, there must be a net power production. The product of density, temperature and confinement time, $\mathrm{nT} \tau$, determines the net power production. According to Lawson's criteria, the product of the density and confinement time for the DD reaction should at least equal $10^{16} \mathrm{~s} / \mathrm{cm}^{3}$ for a net energy output (Serway 1398). High density and confinement allow an increase in the probability of atoms fusing together and releasing energy. With such high temperatures, on the order of one million degrees in the outer region of the plasma, some method other than ordinary materials is needed to confine the plasma to a given volume (Wesson 2).

\section{Tokamaks}

Currently, there are two methods being studied to help achieve higher $\mathrm{nT} \tau$, magnetic confinement and inertial confinement. The magnetic confinement method uses two different magnetic fields to contain the plasma, toroidal and poloidal fields. Since plasmas are affected by magnetic fields and are good conductors of electricity, magnetic confinement is efficient. Most of these magnetic confinement systems use a toroidal device called a tokamak. There are several tokamak experiments worldwide. For instance, the Tokamak Fusion Test Reactor (TFTR) located at the Princeton Plasma Physics Laboratory was designed largely for studies concerning overall net power production, and recently got a world record for $10 \mathrm{MW}$ (megawatts) of fusion power (Wesson 280). The Joint European Torus (JET) in Abingdon, UK is the world's 
largest tokamak. JET studies lie mainly in methods of heating the plasma and energy confinement time, in addition to net power production (Wesson 283). This paper deals with experiments performed on the DIII-D tokamak in San Diego, California. A major part of the DIII-D program is devoted to studying the physics of the edge of the plasma, especially in the divertor regions, and controlling impurities being introduced into the plasma (Mahdavi 1).

DIII-D runs for three weeks at a time with maintenance periods between operations. On a normal operation day, a plasma discharge takes place every fifteen minutes beginning at 9:00 am and ending at 5:00 pm with each discharge lasting for approximately five seconds. It is very important that analysis be done on the shots immediately, so that changes, such as the amount of current in the plasma or an increase or decrease in the pumping out of the neutral gas, can be made, if need be, before the next shot occurs.

Figure 1 shows a schematic of a tokamak. There are two different magnetic fields used in this doughnut shaped device. One is a toroidal field, BT, produced by coils winding around the tokamak. A second magnetic field is the poloidal field, BP, produced by coils, circling the tokamak, and current in the plasma (Serway 1399).

Figure 2 gives a vertical view of the DIII-D tokamak at $240^{\circ}$. It defines some important regions and diagnostics discussed in following sections of the paper.

There are several regions and diagnostics labeled in figure 2. The divertor region, which is of primary concern for this paper, consists of everything below the dotted region as shown in figure 2. Underneath the baffle lies the cryopump, which pumps out unwanted neutral gas particles keeping them from drifting back up into the plasma possibly causing disruptions. Due to the forces of the magnetic fields, particles travel in a helical path eventually striking the walls of the tokamak. After the particles strike the floor, a tremendous amount of heat flux and radiated power comes off the floor as there is a transfer of momentum and energy of particles. As a result, some 
metallic carbon impurities are knocked off the carbon tiles and introduced into the plasma. Inner and outer strike points (ISP, OSP) are labeled in figure 2. These points are possible areas where neutral particles, plasma ions, and electrons might strike the divertor floor.

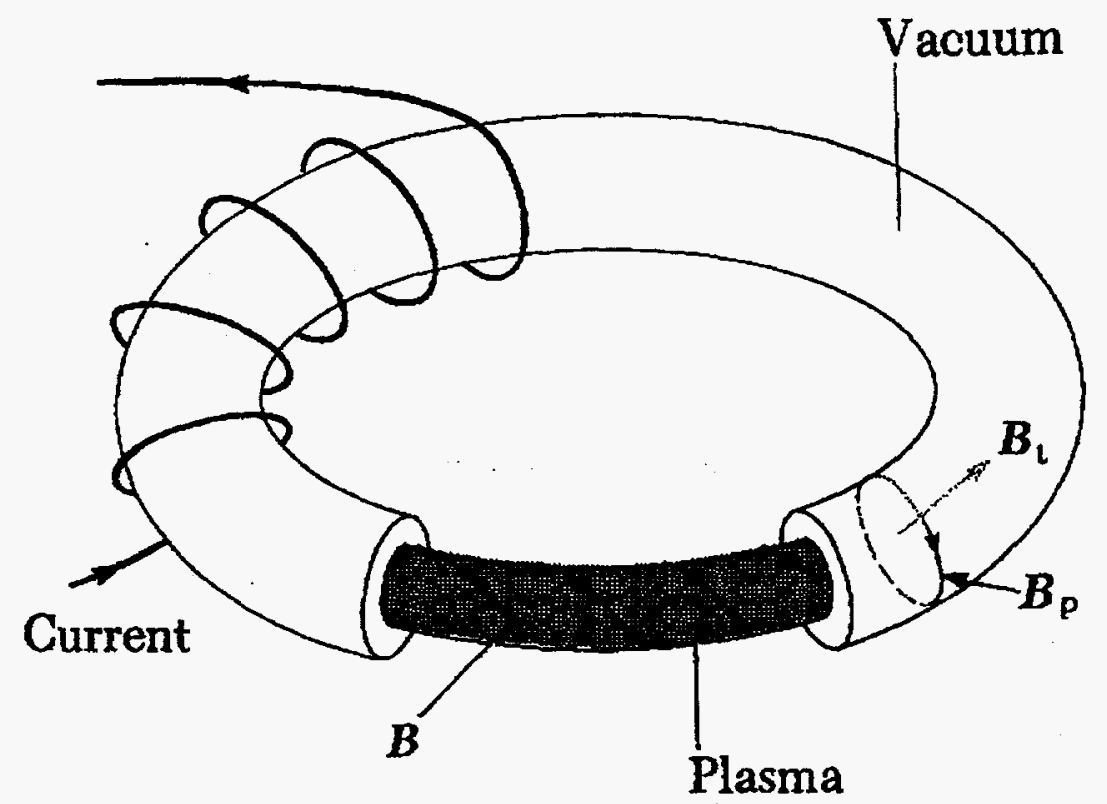

Figure 1: The primary magnetic field is the toroidal field. Current passes through the primary coil; which produces a change in flux through the torus. The change in flux results in a toroidal electric field, which drives a current through the plasma. The resulting magnetic field is helical around the plasma, which helps to confine it within the tokamak walls (Wesson 12).

\section{Bolometers}

There are several ports located along the walls of the tokamak as shown in figure 2. These ports contain different diagnostics such as infrared cameras, photo diodes, and bolometers. Bolometers are devices measuring radiated power profiles within DIII-D. The majority of the measured radiated power comes from atoms emitting photons going from one ionization state to another, or emissions from neutral atoms going from excited states to ground states. When particles strike the divertor floor or 
walls, energy is transferred in the form of heat to the carbon tiles lining the tokamak. A tiny fraction of the total radiated power measured by the bolometers comes in the form of infrared radiation from the hot carbon tiles. Studies are being done to develop ways of dissipating the heat flux to the divertor floor, possibly by keeping particles from striking the floor. Measurements of the radiated power are necessary to assess applicability of techniques, such as gas puffing, to lessen this heat flux to the floor (Mahdavi).

\section{Bolometer Diagnostic}

\section{Sensors}

Figure 3 is a diagram of a bolometer interceptor. Each bolometer interceptor has two sensors, a reference sensor and an active sensor, made up of several layers. Mounted beside one another, these two sensors are on an alumina block $0.64 \mathrm{~cm}$ wide, $3.02 \mathrm{~cm}$ long, and $0.32 \mathrm{~cm}$ thick. Under the active sensor, a cut keeps the sensor thermally isolated from the substrate. A cover piece, made of a polymide, Vespal, known for its strength, vacuum capability, and high temperature characteristics, is placed over the sensors to protect them from possible plasma radiation. The sensors have a maximum allowable temperature of $200^{\circ} \mathrm{C}$, and cooling is provided for them during a high temperature bake $\left(350^{\circ} \mathrm{C}\right)$ of DIII-D. There is an opening in this cover piece over the active sensor exposing it to incoming radiation (Leonard 3). Light radiation strikes platinum foil, the first layer, where it heats the foil. Under the foil is a layer of electrical isolation material called Kapton. A platinum wire connected to gold plated pins lies underneath the Kapton. As the wire heats, it's voltage as well as current changes resulting in a change in resistance, $R=V$ /1. The power signal is calculated using the time rate of change of the resistance (Leonard 7). 


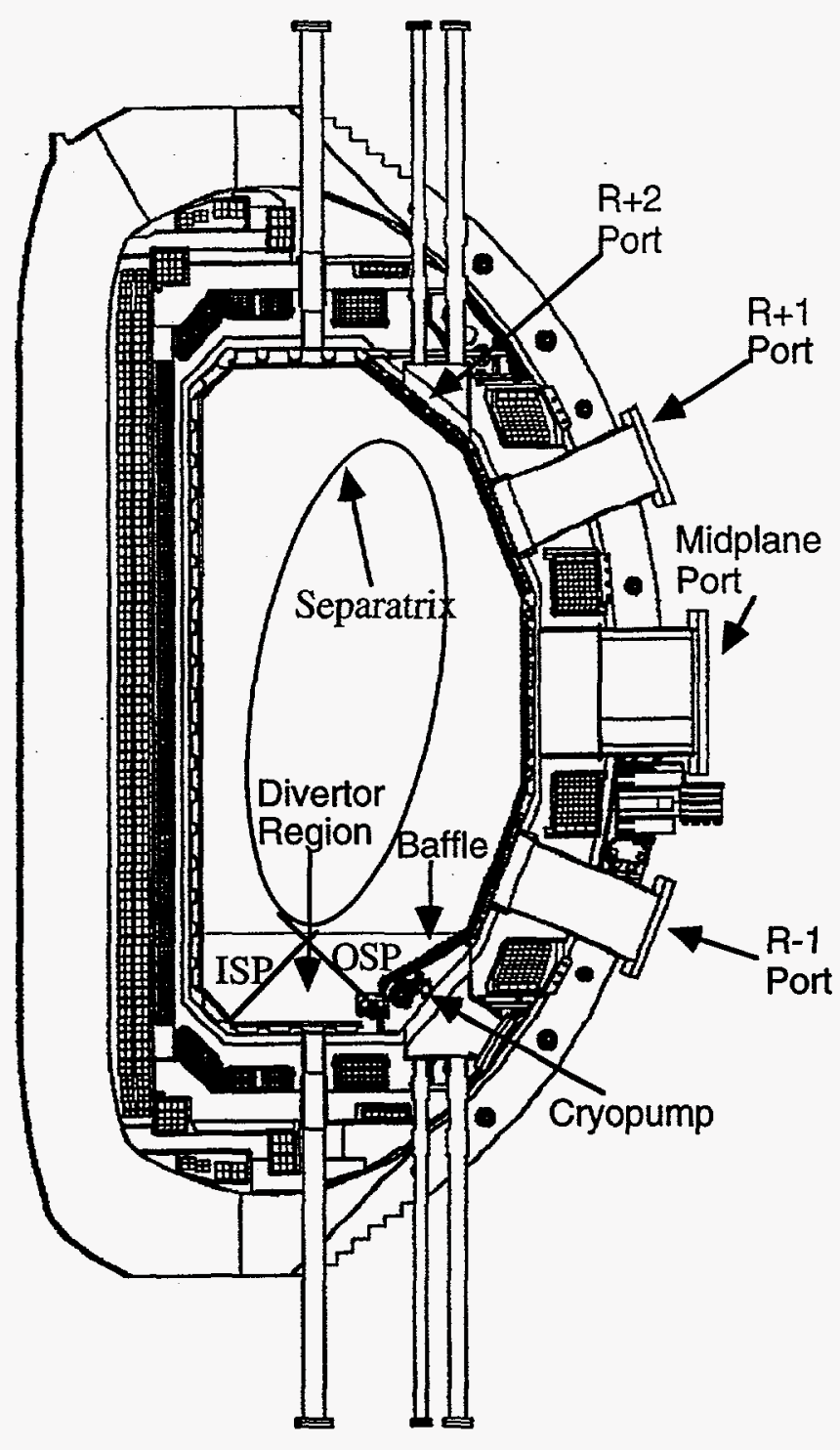

SECTION AT 240

Figure 2: A vertical slice located at $240^{\circ}$ for the DIII-D tokamak.

\section{Geometry}

There are a total of twenty-four interceptors in each bolometer diagnostic.

These interceptors are enclosed in copper housings which have an aperture for viewing the plasma. For one bolometer diagnostic, two housings are needed to 
achieve a full view of the plasma. Each housing contains roughly half of the twentyfour interceptors (Leonard 3). There are two bolometer diagnostics in the DIII-D tokamak one located in port $R+2$ and one in port R-1. Therefore, a total of forty-eight bolometer channels and viewing chords exist. These viewing chords are pictured in figure 4. The diagnostic in port $R+2$ gives the vertical view of the plasma, and the diagnostic in port R-1 gives the horizontal view. These two views can be combined to get a two dimensional radiated power profile of the plasma.

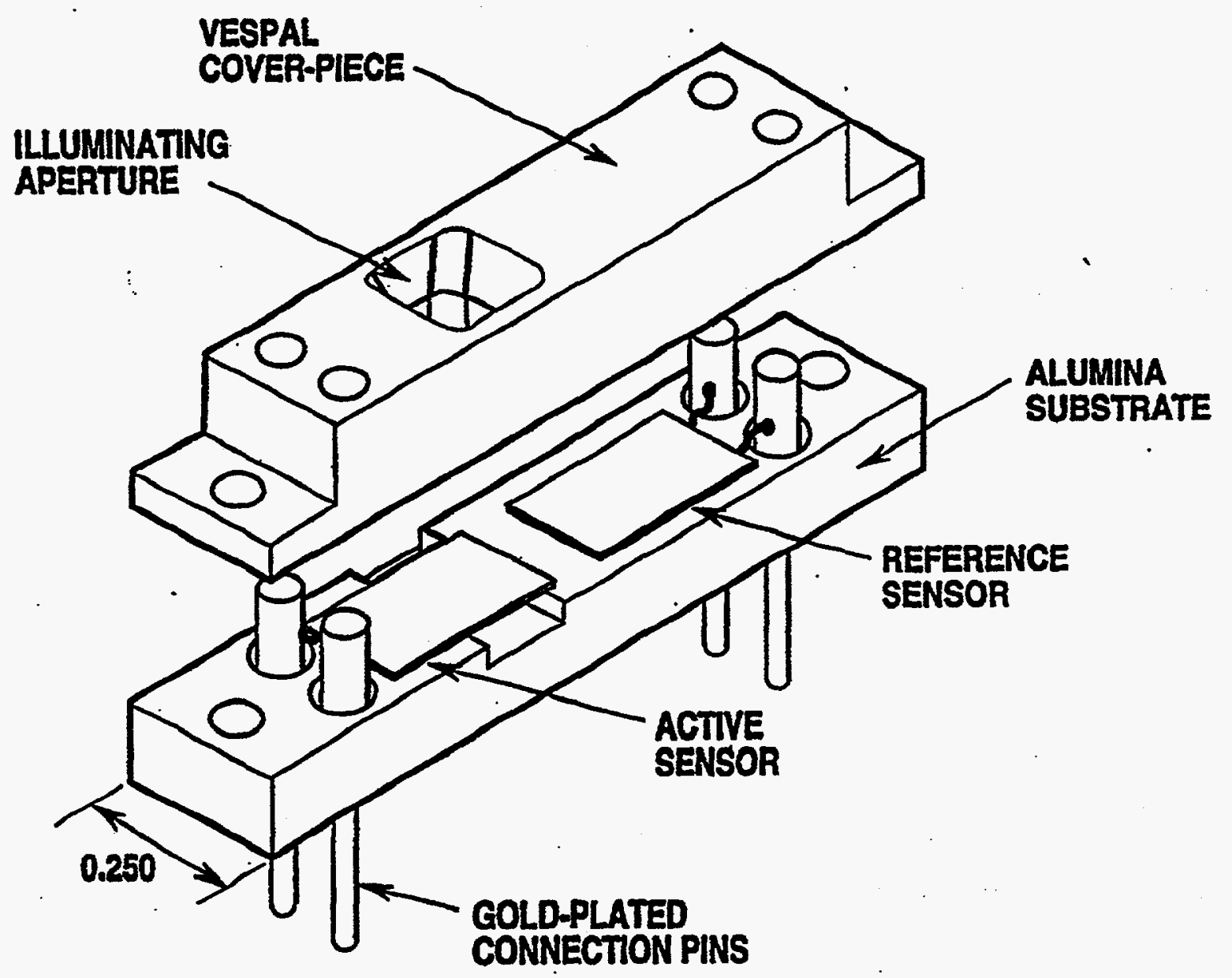

Figure 3: A bolometer interceptor (Leonard 4). 


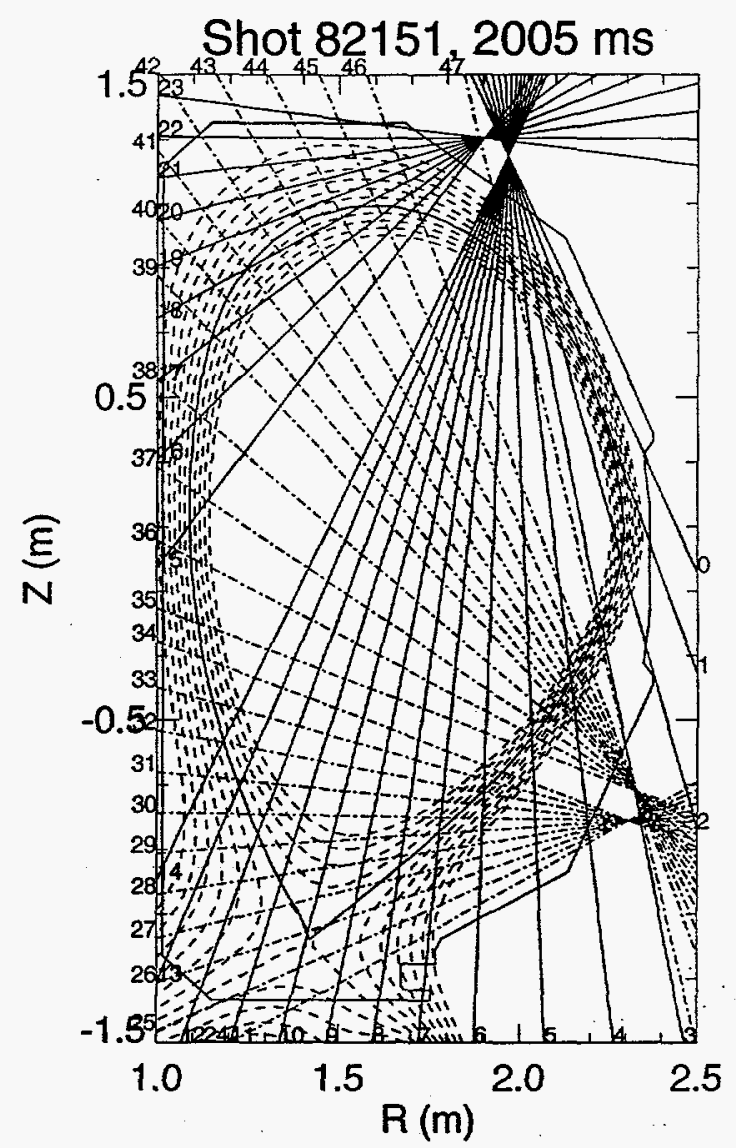

Figure 4: Bolometer viewing chords.

\section{Old versus New Arrays}

The two diagnostics located in ports $\mathrm{R}+2$ and $\mathrm{R}-1$ are additions to the DIII-D tokamak. Previously only one bolometer diagnostic located in the midplane port ( shown in figure 2 ) existed. However, it was found that this one diagnostic was not adequate for determining the power radiated in the divertor region. The viewing chords for this region were too large to give a good representation of the divertor region. The mid plane diagnostic also captured power radiated from the core. With only the one diagnostic the geometry for the viewing chords was not accurate, and the power radiated readings were not well understood. The new bolometer diagnostics 
provide better spatial resolution in the divertor region and have complete plasma coverage (Leonard 4).

\section{UEDGE Code}

\section{Purpose}

Since LLNL assists General Atomics with DIII-D data analysis, they also do simulations using a code called UEDGE. The UEDGE code is a Unified Tokamak Edge Physics Modeling Code. Its main interest lies in the edge regions of the plasma formed in a tokamak. Its purpose is to model the plasma physics involved during a fusion reaction simulation. The code calculates quantities such as electron and ion density and temperatures, as well as radiated power (UEDGE Manual).

\section{How UEDGE Works}

Using fluid equations and different boundary conditions for the core and outer or inner wall divertor regions, the UEDGE code models the physics of the plasma created within the scrape-off-layer of a tokamak. UEDGE has its own two-dimensional radial and poloidal grid as shown in figure 5 below. This grid consists of an inner region, a separatrix, refer to figure 2 , and a scrape off layer (SOL). The UEDGE code can model physics in these regions during ionization, recombination, and impurity radiation. The physics models contained in the UEDGE code must be validated against experimental data before the code is used to aid in the design of future experiments. It is hoped that much will be learned from these UEDGE simulations, and then applied to experimental runs with expectations of getting closer to the goal of developing an efficient commercial fusion reactor. 


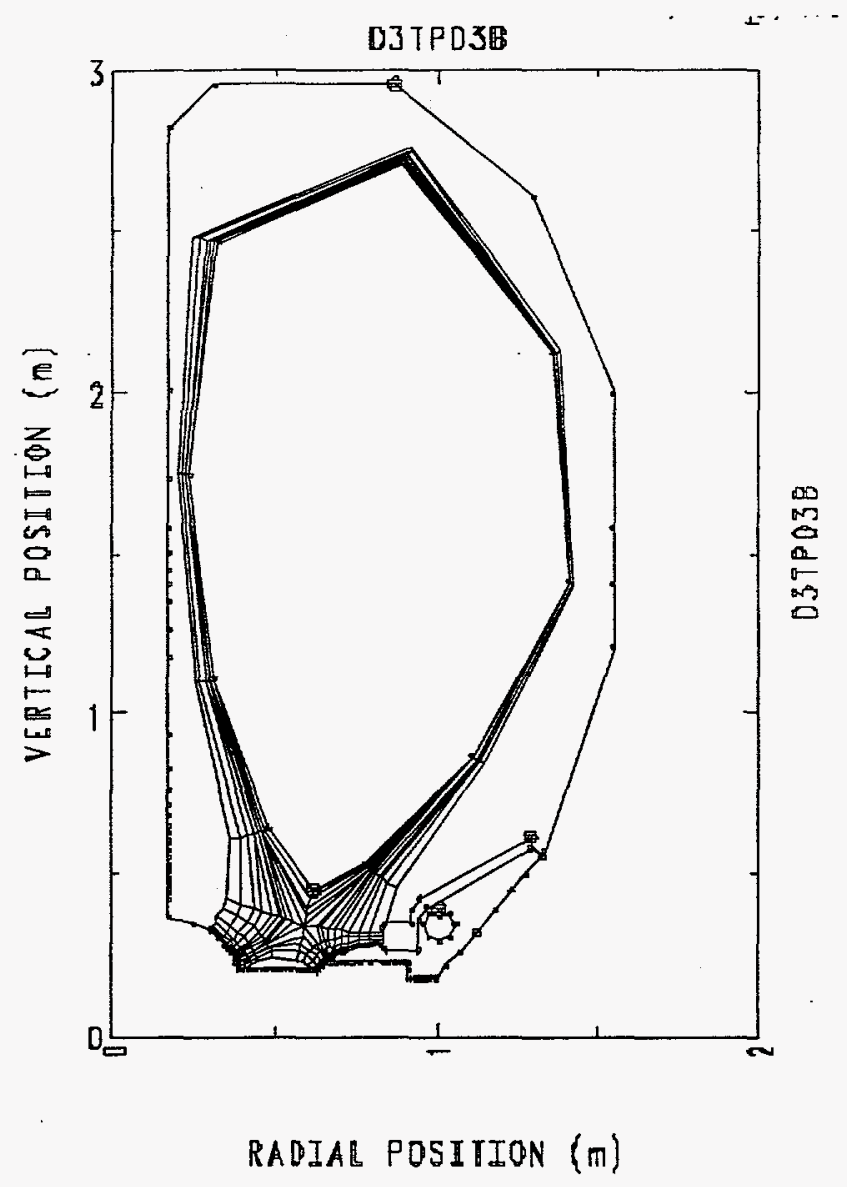

Figure 5: UEDGE grid

\section{Radiated Power Calculations by UEDGE}

From the UEDGE grid, the code is able to calculate the radiated power in a toroidally continuous volume represented in the poloidal cut by a UEDGE grid cell.

\section{Bolometer Post Processor}

\section{Purpose}

A bolometer post processor was developed to obtain volumetric radiated power values as calculated by UEDGE, and convert them into line integrated radiated power along bolometer lines of sight. From these values, a plot is made of the channel number versus radiated power to the distribution of the radiated power. These 
calculated values are then compared with actual bolometer experimental data taken from DIII-D in San Diego. These comparisons help to identify which of the UEDGE model assumptions best represent the physics occurring in DIII-D experiments.

\section{How Processor Works}

UEDGE is a plasma physics code which runs under the Basis system. UEDGE calculates plasma physics quantities and stores the values in a BASIS database. The post processor accesses these values from the database and within the BASIS system performs calculations with them, eg. line integrals of the radiated power (Dubois 2).

\section{Preparation of UEDGE Data}

The bolometer Post Processor, after it has loaded in all the appropriate variables, takes the volumetric UEDGE radiated power values, converts them into an orthogonal $\mathrm{RZ}$ grid, and then integrates along the bolometer lines of sight of the bolometer chords to get a calculated line integrated radiated power. This data is then written to a file called "bolo_probname", where probname is the name of the UEDGE problem being run. After this data is obtained, actual bolometer data is received using codes written in the Interactive Data Language, IDL (IDL Reference Guide). Bolometer data is extracted from the DIII-D experimental database and put into tabular form (Preckshot). Both sets of data, UEDGE solutions and actual data, must be in a tabular format before they are read into a spreadsheet program. Once the data is placed in a spreadsheet program, comparison graphs are made and analysis done.

\section{Analysis of Shot 82151 and Comparisons to UEDGE Simulations}

Shot 82151 is a DIII-D experimental shot lasting for 5 seconds. The review plot in figure 6 gives a summary of this particular shot. This shot was done to study the effects of injecting gas up into the plasma from the divertor floor in order to decrease 
the heat flux to the floor. We were looking for a decrease in the divertor power arising from an increased total radiated power (see last graph in figure 6). The total current used in this shot was 1.62 MA (megaamps) with a magnetic field, BT, equal to $2.01 \mathrm{~T}$ (tesla). An initial deuterium puff began the discharge with another deuterium injection around $2100 \mathrm{~ms}$ (milliseconds). Our analysis of this shot takes place at $2005 \mathrm{~ms}$, right before the second deuterium puff.

\section{1}

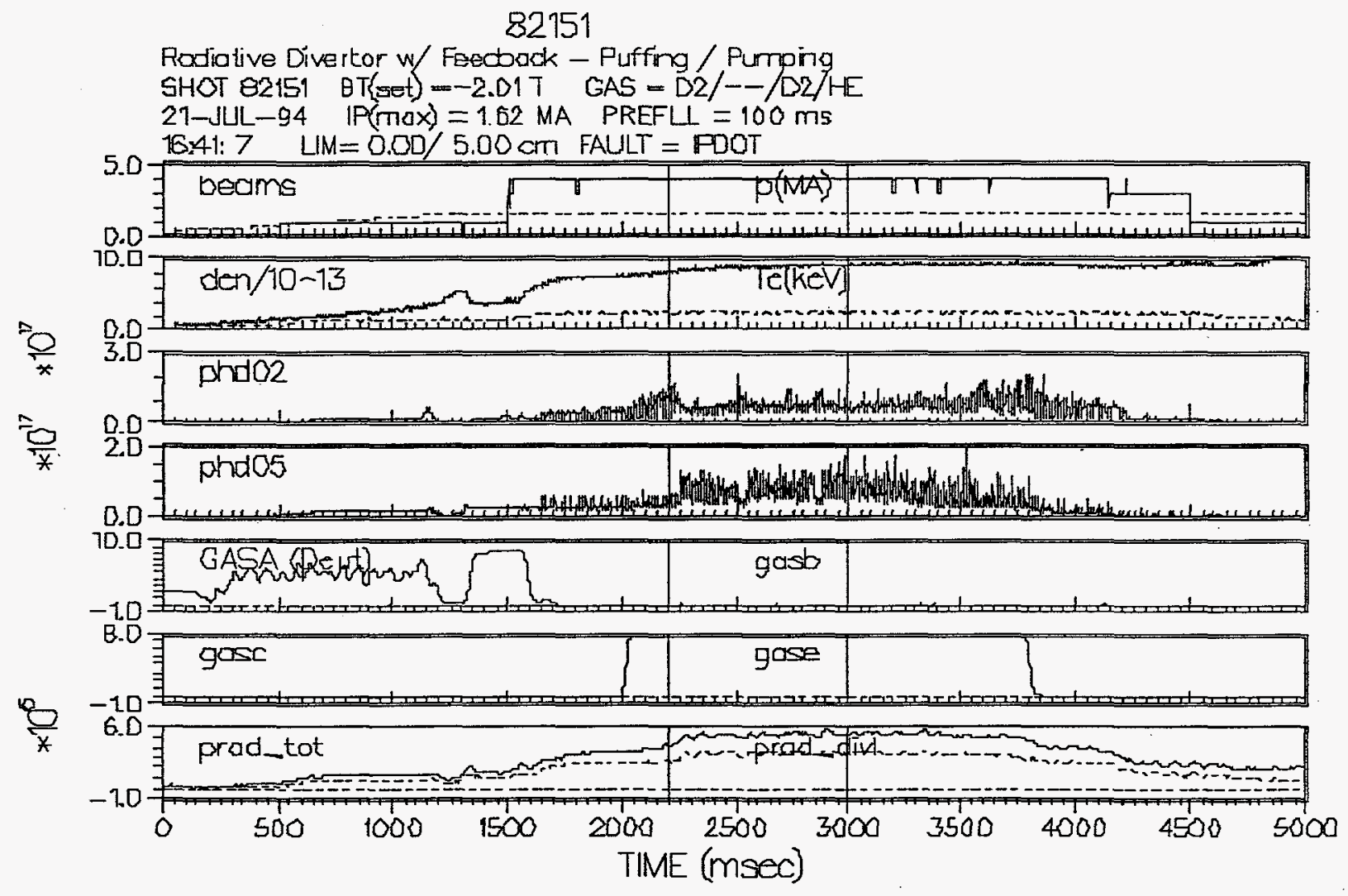

Figure 6: A review plot shows the plasma current, Ip, injected beam power, beams, deuterium gas injection, GAS A and gase, line averaged density, den / 10^13, line integrated $\mathrm{H}_{\alpha}$ emission, phd02 and phd05, electron temperature, $\mathrm{Te}(\mathrm{keV})$, total radiated power, prad_tot, and outer divertor power, prad_div. 


\section{No Impurity Radiation}

The first UEDGE simulation compared to shot 82151 , involves only radiation from the hydrogen species. No impurities are included in this simulation. Therefore, this simulation could possibly be leaving a lot out, since we know there are impurities introduced in actual DIII-D shots. Figure 7 shows the plot comparisons. Channel numbers seven and eight from the upper array give a view of the outer strike point radiated power. Channel numbers eleven and twelve from the upper array give the inner strike point power, which is a factor of nearly three greater than the outer strike point power. Channel numbers twenty-four and twenty-five give another view of the inner strike point as seen from the lower bolometer array. At this point the magnitude is less than the actual data. In channel numbers thirty-five and thirty-six we see some power being radiated from the inner wall area. What is important about the results from the UEDGE simulation is not magnitudes of the power, but how well the simulations line up with the actual data as far as the channel numbers. For this no impurity simulation, the curves for the peaks match fairly well.

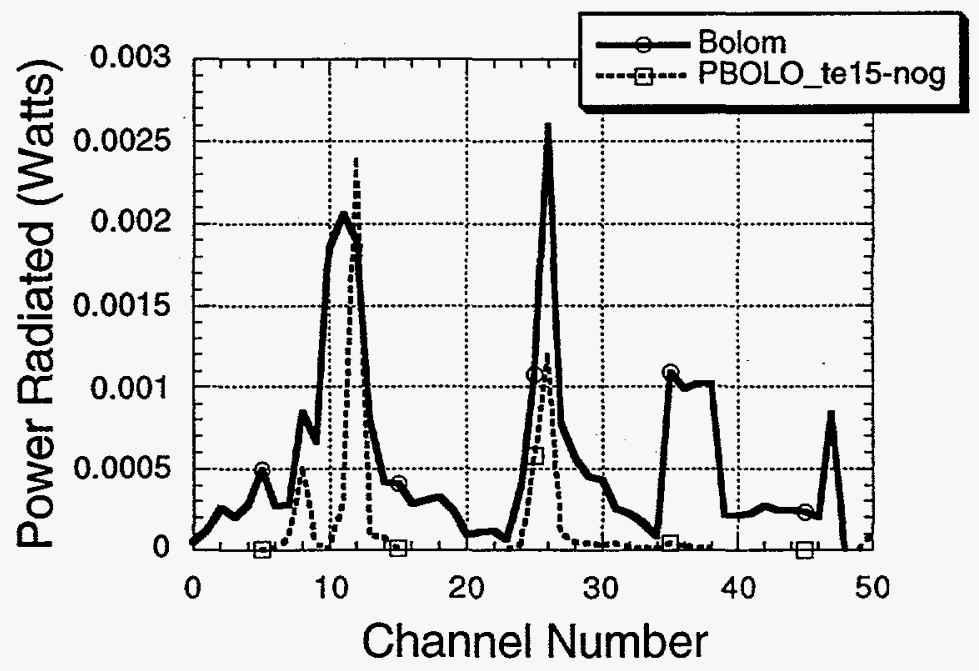

Figure 7: A comparison of Shot 82151 at $2005 \mathrm{~ms}$ with a UEDGE simulation where hydrogen is the only contributor to the radiated power. No impurity radiation exists in this UEDGE simulation. 


\section{Constant Concentration of Impurities}

This UEDGE simulation assumes a constant concentration of average Carbon impurities. This simulation ensures constant concentration by specifying the density of Carbon divided by the density of the electrons as the same throughout the UEDGE plasma grid. While there is no reason, a priori, to expect a constant density fraction, this model is a simple way of introducing impurity radiation into the UEDGE simulation. In the cases with constant impurity concentration and with the impurity transport model (see following paragraph), the amount of Carbon impurities introduced is adjusted to match the total calculated radiated power to the measured value. That is, an amount of Carbon impurities is introduced into the simulation until the total radiated power matches that of the DIII-D shot 82151 total radiated power. In the constant concentration model the Carbon impurities are introduced as a fixed fraction of the electron density everywhere, while in the impurity transport model, carbon is introduced uniformly around the SOL of the UEDGE grid. Figure 8 shows this comparison. The simulation, in terms of curve matching, is not a good match to the actual DIII-D data. The peaks for the inner and outer strike points are the only regions in the graph where a good match exists. Similar to the no impurity radiation model, channel numbers eight and nine in figure 8 represent the outer divertor leg peak. Channel number eleven shows the inner divertor leg peak as seen from the upper viewing array. Channel number twenty-five shows a view from the lower array of the inner divertor leg radiated power. No other channel numbers give much, if any, radiated power values. 


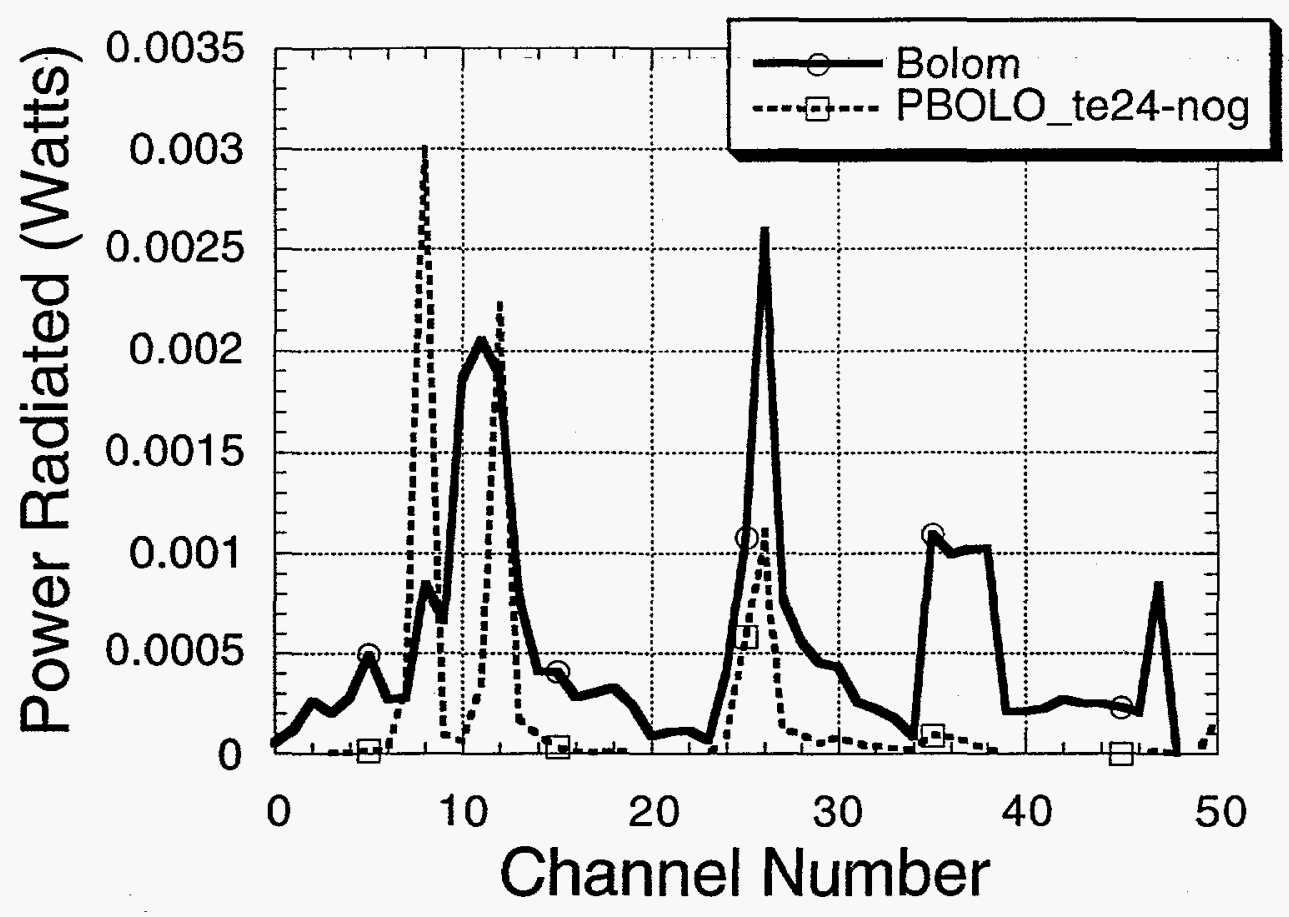

Figure 8: A plot comparison of Shot 82151 at $2005 \mathrm{~ms}$ and a UEDGE simulation assuming a constant concentration of impurities.

\section{Impurity Transport}

The final impurity model used in UEDGE takes into account all forces, especially plasma flows and temperature gradients, to determine the impurity density. Figure 9 shows a comparison between the data and the simulation. The radiated power peaks at the inner and outer strike points from the upper array in channel numbers eight and nine for the outer divertor leg and channel number eleven for the inner divertor leg. The inner strike point from the lower array shows a peak at channel number twenty-five. Some power is picked up from the inner wall around channel numbers thirty-five and thirty-six. Between the divertor leg peaks, some additional power signals are picked up similar to the actual data. This impurity model gives us the best fit to the actual bolometer data. 


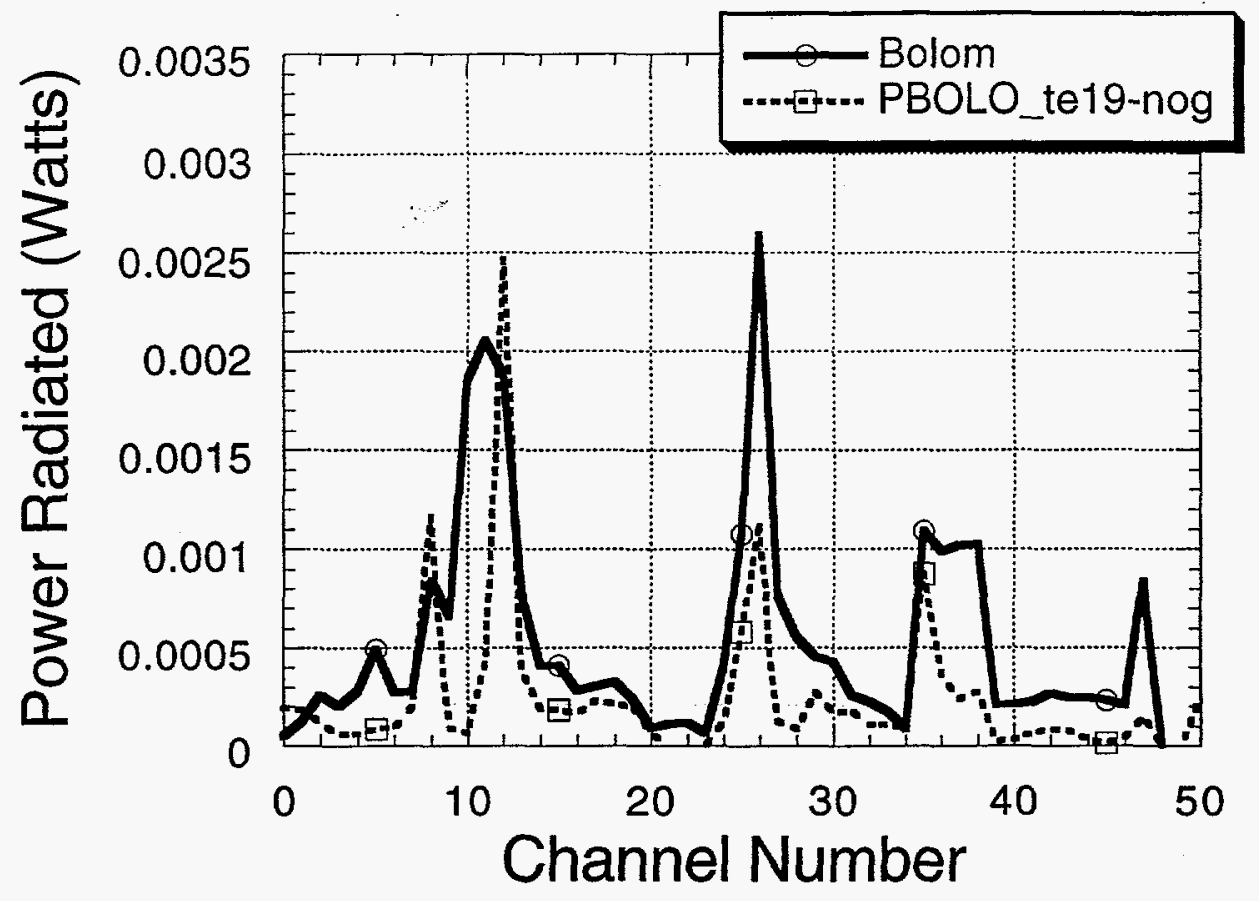

Figure 9: A comparison plot of Shot 82151 at 2005 ms with a UEDGE simulation with a fractional concentration of impurities.

\section{Conclusion From Comparisons}

My research has been completed by developing the bolometer post processor. This post processor can now be used as a tool for comparing data from UEDGE simulations against DIII-D bolometer data. Previously, no ways existed to compare the simulations against actual bolometer data. Now corrections can be made to UEDGE plasma simulations to help improve the models used.

\section{Impurity Model Needed}

The no impurity model with only hydrogen radiation shows large differences from the actual DIII-D data. The existing peaks come about only from the divertor floor inner and outer strike points. No other similarities in the data exist. This model does 
not adequately reproduce the actual data. This comparison justifies that some impurity model is needed for UEDGE plasma simulations.

\section{Carbon Impurity Introductions}

In the constant concentration model, the impurities lie in the region of highest density, which is near the plates in the divertor region. As a result, we see in figure 8 the large inner and outer strike point peaks. This constant concentration model appears to be poor because of the way impurities are introduced into the simulation, as a fraction of the electron density.

\section{Physics of Carbon Migration}

The introduction of Carbon uniformly around the outer SOL, with the transport model, appears to be a better model, since the physics of Carbon migration pulls the Carbon down to the divertor floor. It is assumed that $90 \%$ of the Carbon is then recycled, and a build up of Carbon begins to occur at the divertor floor. The transport model then spreads this Carbon throughout the divertor legs where it radiates. This cools the plasma, reducing the hydrogenic radiation so that the sum near the strike points still matches the measured power for the chords. With a lower density of Carbon at the divertor floor, we get better matches to the bolometer data at the inner and outer strike points than in the constant concentration model.

Before any other conclusions are made, we are increasing the resolution of the grid on which the line integrals of the UEDGE power are calculated, to see if this affects the results. This and further analysis will be reported at a future date. 


\section{Summary}

My bolometer post processor obtains volumetric radiated power values calculated by UEDGE, and converts them into line integrated radiated power along bolometer lines of sight. From this data, plots are made of the bolometer channel number versus the radiated power along the bolometer lines of sight. These simulated values are compared to actual DIII-D bolometer data. This post processor can now be used for bench marking the physics models used in UEDGE plasma simulations. The first use of the bolometer post processor was to validate the average ion impurity model used in UEDGE. Three power radiation examples were examined to determine the impurity model's accuracy. It is not important, at this stage, that the graphs lie on top of each other, but the curves should at least have the same form. The preliminary conclusion from this comparison is that the impurity transport model gives the best match to the data, and both the no impurity, hydrogenic radiation only, and uniform concentration models give substantially poorer agreement with the data. This bolometer post processor will be used in the future to determine the accuracy of other UEDGE models.

\section{Acknowledgments}

This work was performed under the auspices of the U.S. Department of Energy by Lawrence Livermore National Laboratory under contract number W-7405-ENG-48, also by General Atomics under contract number DE-AC03-89ER51114. I would like to thank my two mentors Max Fenstermacher and Gary Porter for all their help and understanding during my research. I wish to thank Bill Meyer and Gary Porter for performing computer calculations with their codes so analysis could be done. I would like to thank Tom Casper, Ray Jong, and Mike Brown for their computer support, Ron Ellis for the graphics pictures, Charlie Lasnier for his help during my stay at General Atomics, Tony Leonard for his help in describing bolometer design and function, and Marv Rensink and Thomas Rognlien for their valuable suggestions. 


\section{Reference List}

Dubois, P. F., Motteler, Z. C., Willmann, P. A., et al. The Basis System. U.S.

Department of Energy: Regents of the University of California, March 26, 1990. 2-15.

IDL Reference Guide. Version 3.5. Research Systems, Inc., Boulder, CO. November 1993.

Leonard, A. W., Meyer, W. H., Geer, B., Behne, D. M., Hill, D. N. 2-D Tomography with Bolometry in DIII-D. General Atomics, July 1994. This is a preprint of a paper to be presented at the Tenth Topical Conference on High Temperature Plasma Diagnostics, May 8-12, 1994. Rochester, New York and to be printed in Review of Scientific Instruments.. Work supported by: U.S. Department of Energy under contract Nos. DE-AC03-89ER51114 and W-7405-ENG-48. General Atomics Project 3466.

Mahdavi, M. A., et al. Divertor Heat and Particle Control Experiments on the DIII-D Tokamak. General Atomics, July 1994. This is a preprint of a paper to be presented at the Eleventh International Conference on Plasma Surface Interactions in Controlled Fusion Devices, May 23-27, 1994, Ibaraki-ken, Japan, and to be printed in the Proceedings. Work supported by: U.S. Department of Energy under contract Nos. DE-AC03-89ER51114, W-7405-ENG-48, DE-AC0584OR21400, DE-AC03-76DP00789, and Grant No. DE-FG03-89ER51121. General Atomics Project 3466. 
Preckshot, G. G., Butner, D. N., Brown, M. D., Meyer, W. H. Transpaerent Data Access in a Multi-vendor. Distributed Data Acquisition and Data Processing System. 7th Top. Conference High Temperature Plasma Diagnostics, Napa, CA, March 13-17, 1988.

Serway, Raymond A. Modern Physics Supplement to Accompany Physics for Scientists and Engineers. 3rd ed. Philadelphia, Fort Worth, Chicago, San Francisco, Montreal, Toronto, London, Sydney, Tokyo: Saunders Golden Sunburst Series, Saunders College Publishing, 1990. 1394-1400.

UEDGE Manual. The Regents of the University of California, 1994. This work was produced at the University of California, Lawrence Livermore National Laboratory (UC LLNL) under contract no. W-7405-ENG-48 (Contract 48) between the U.S. Department of Energy (DOE) and The Regents of the University of California (University) for the operation of UC LLNL. Copyright is reserved to the University for purposes of controlled dissemination, commercialization through formal licensing, or other disposition under terms of Contract 48; DOE policies, regulations and orders; and U.S. statutes. The rights of the Federal Government are reserved under Contract 48 subject to the restrictions agreed upon by the DOE and University as allowed under DOE Acquisition Letter 88-1.

Wesson, John. Tokamaks. eds. A. Acrivos, F. W. Crawford, L. C. Woods, J. M. Brady, A. L. Cullen, C. P. Wroth. New York: Oxford University Press, 1987. 